\title{
The ATLAS Analysis Architecture
}

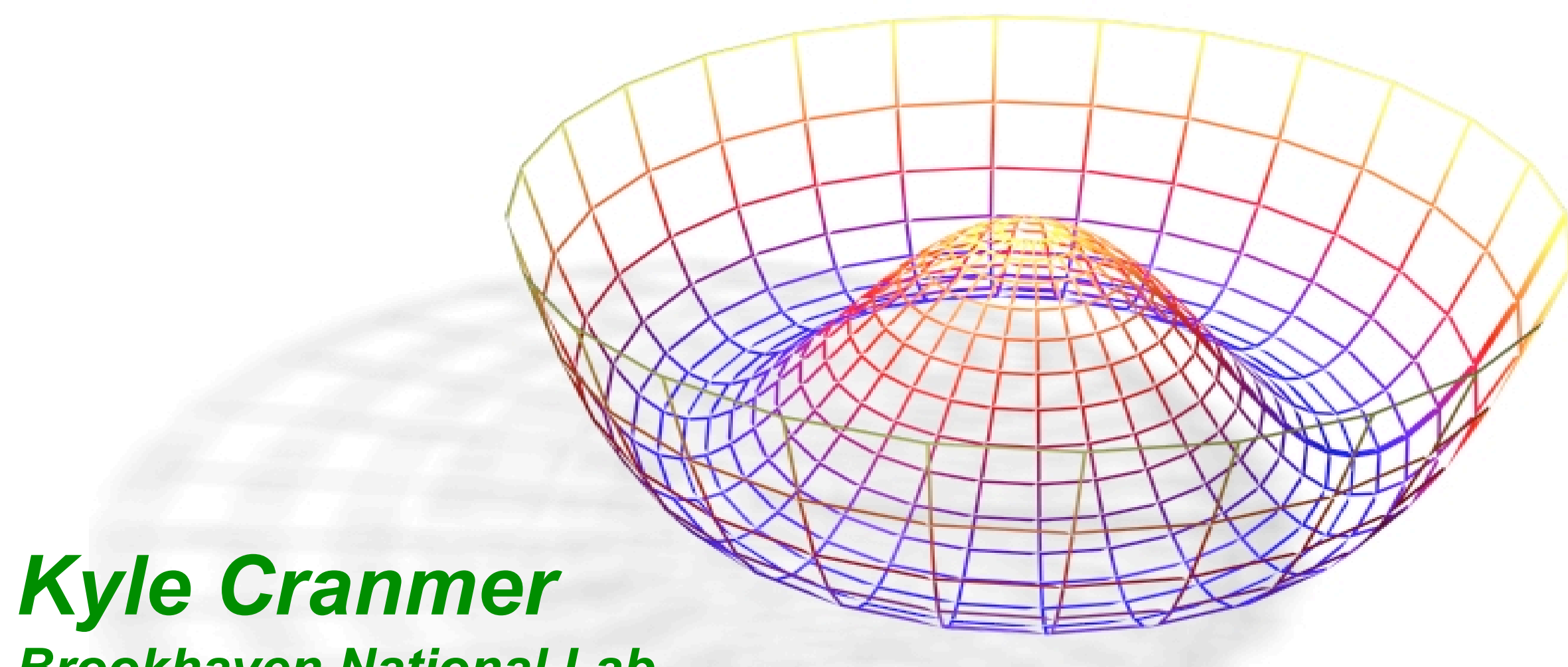

Brookhaven National Lab 


\section{Introduction}

I will review the ATLAS analysis architecture including:

- The data chain from trigger to final analysis results

- The key architecture components and design principles

- The analysis tools and distributed analysis on the grid

- The recent developments in our analysis model

I will try to present this from a physicist point of view instead of a computing point of view 


\section{The ATLAS Experiment}

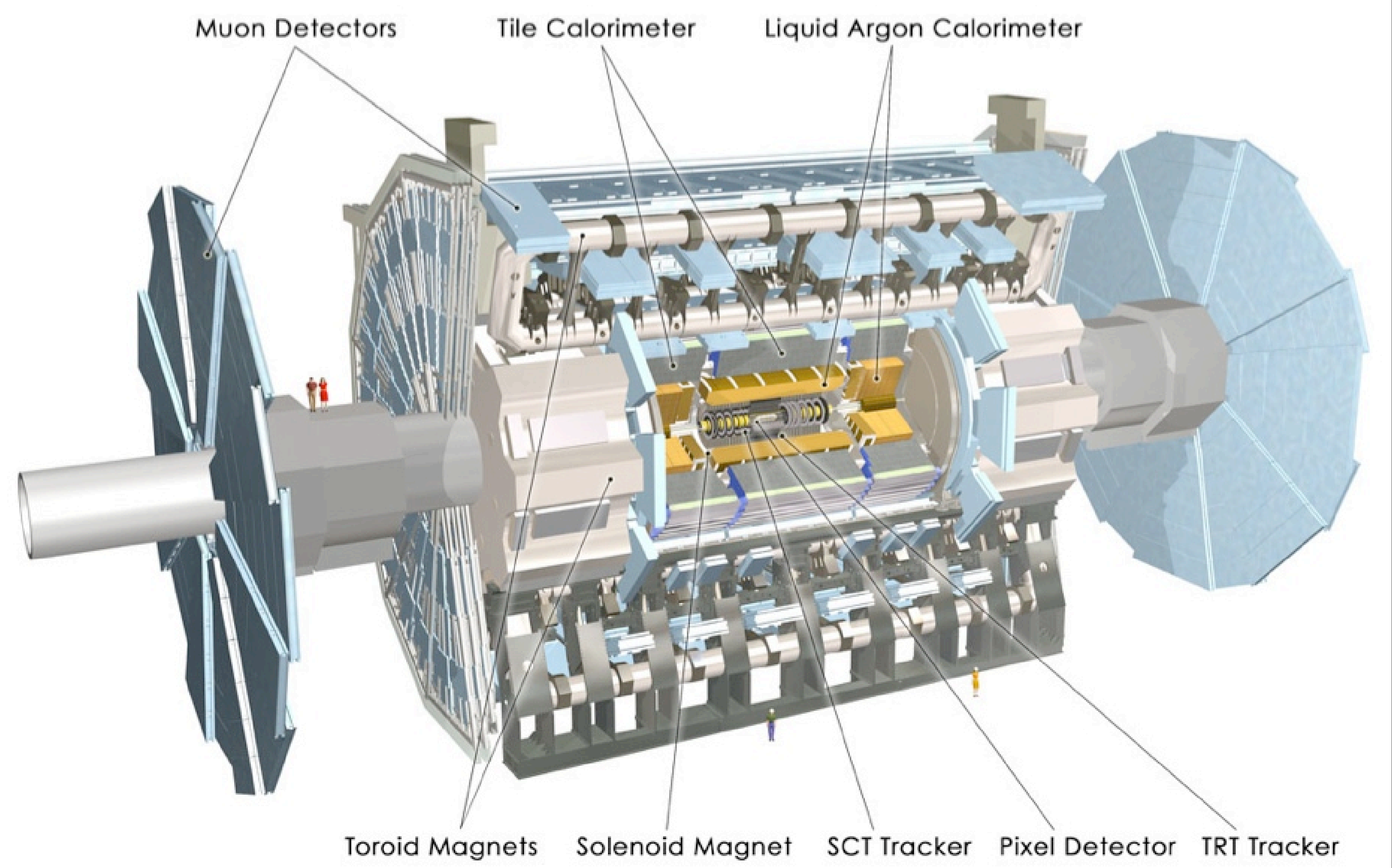




\section{The ATLAS Experiment}

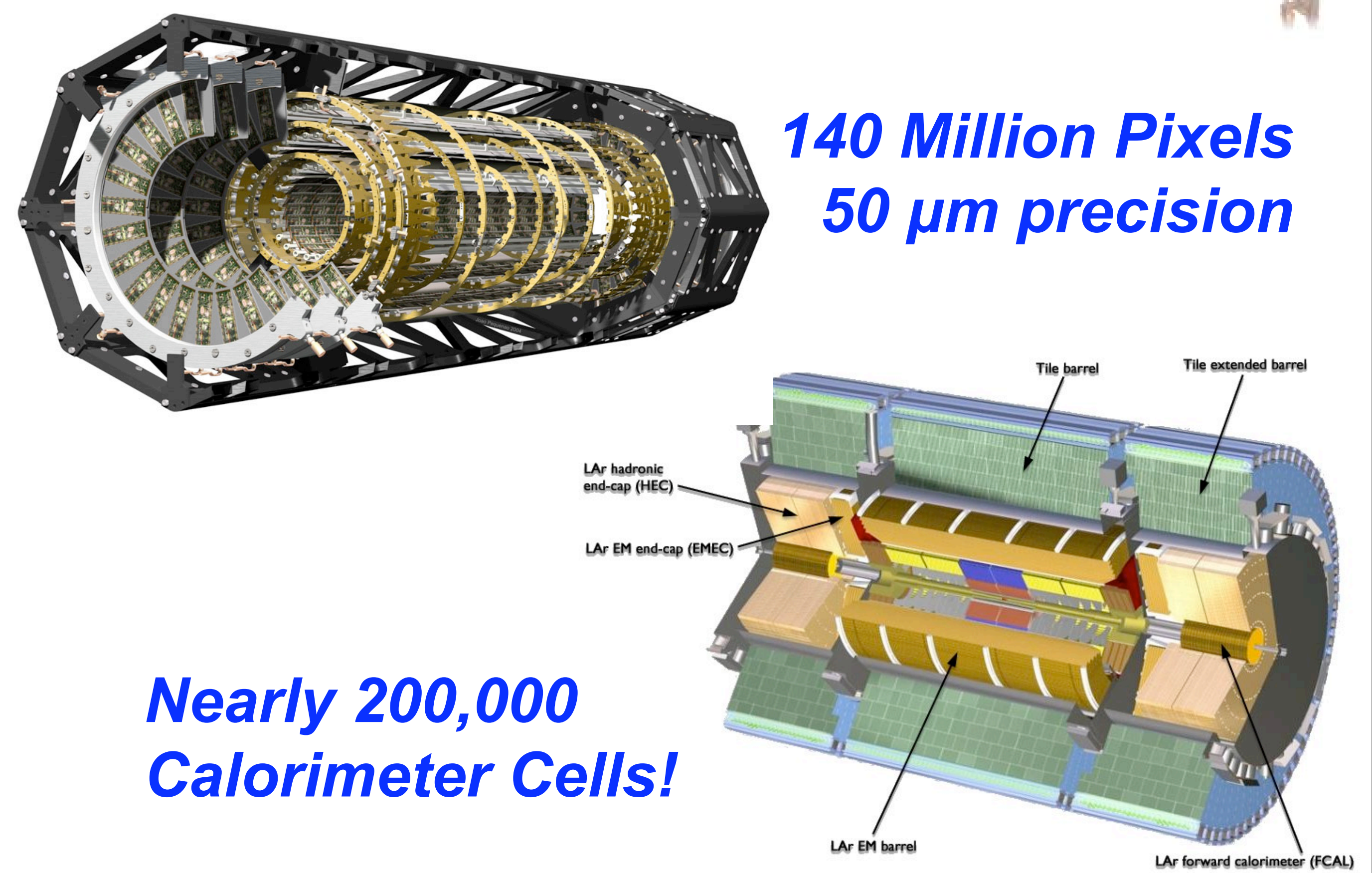




\section{Triggering}

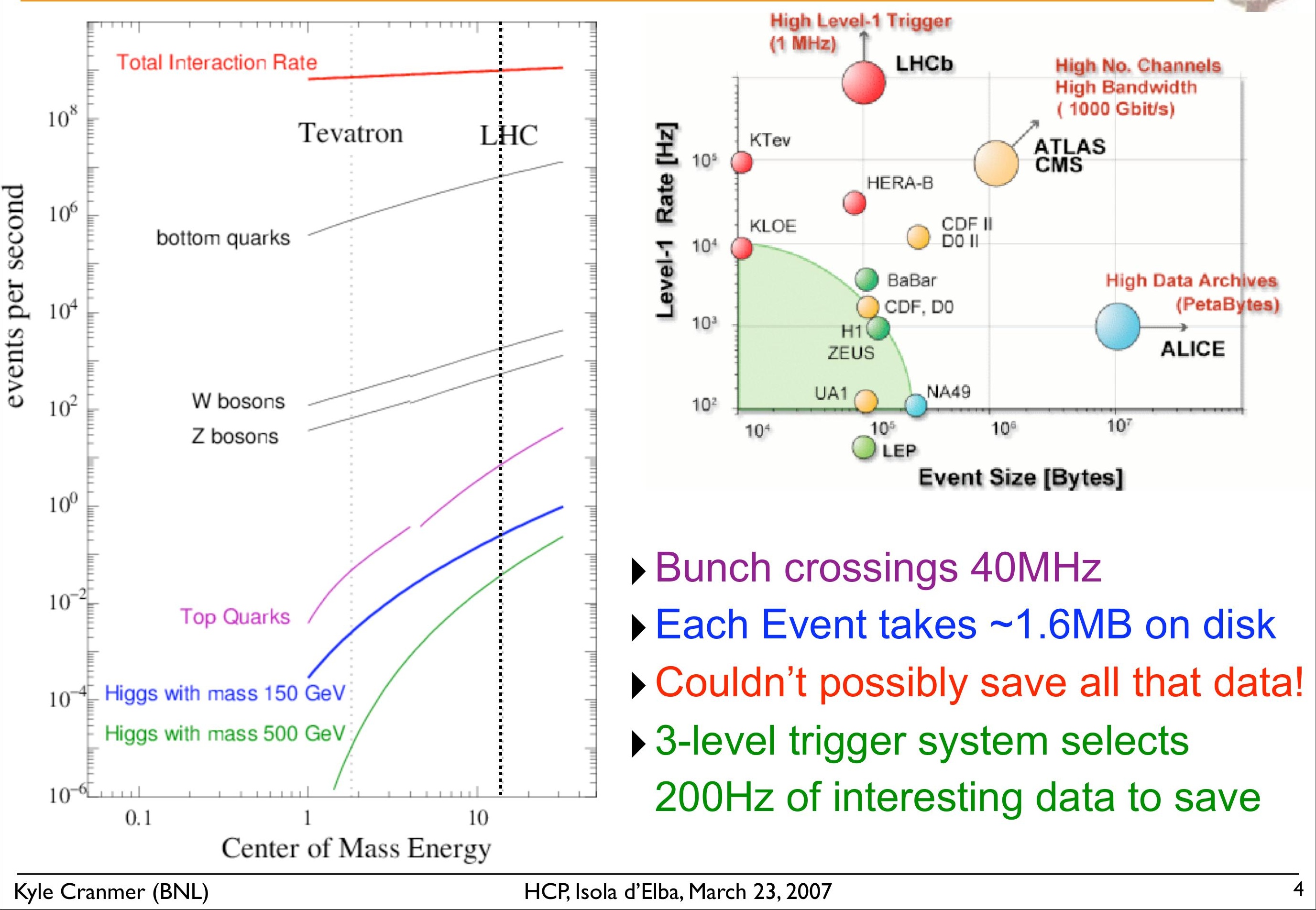




\section{Trigger \& Data Acquisition}

\section{$\underline{\text { Trigger }}$}

$40 \mathrm{MHz}$

$75 \mathrm{kHz}$

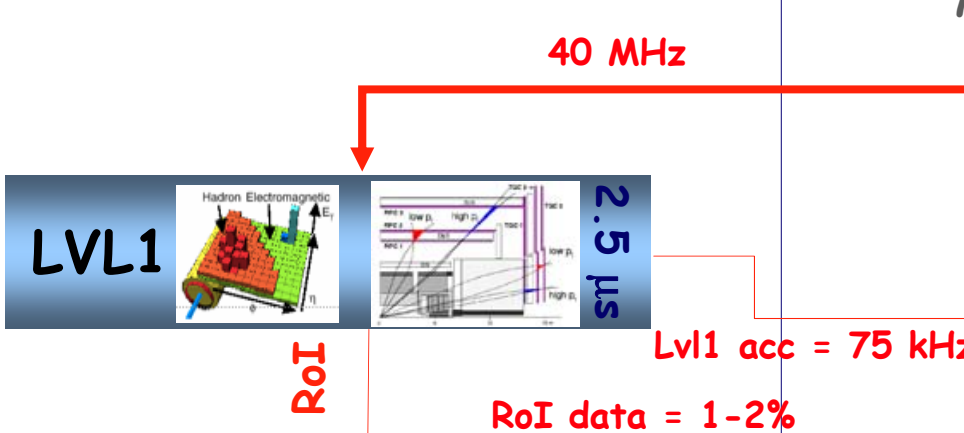

Calo

DAQ

MuTrCh Other detectors

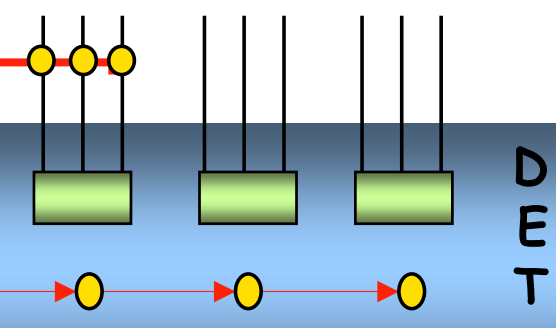

FE Pipelines

\begin{tabular}{|l|l|}
\hline ROD ROD ROD \\
\hline
\end{tabular}

|| 120||$G B / s$

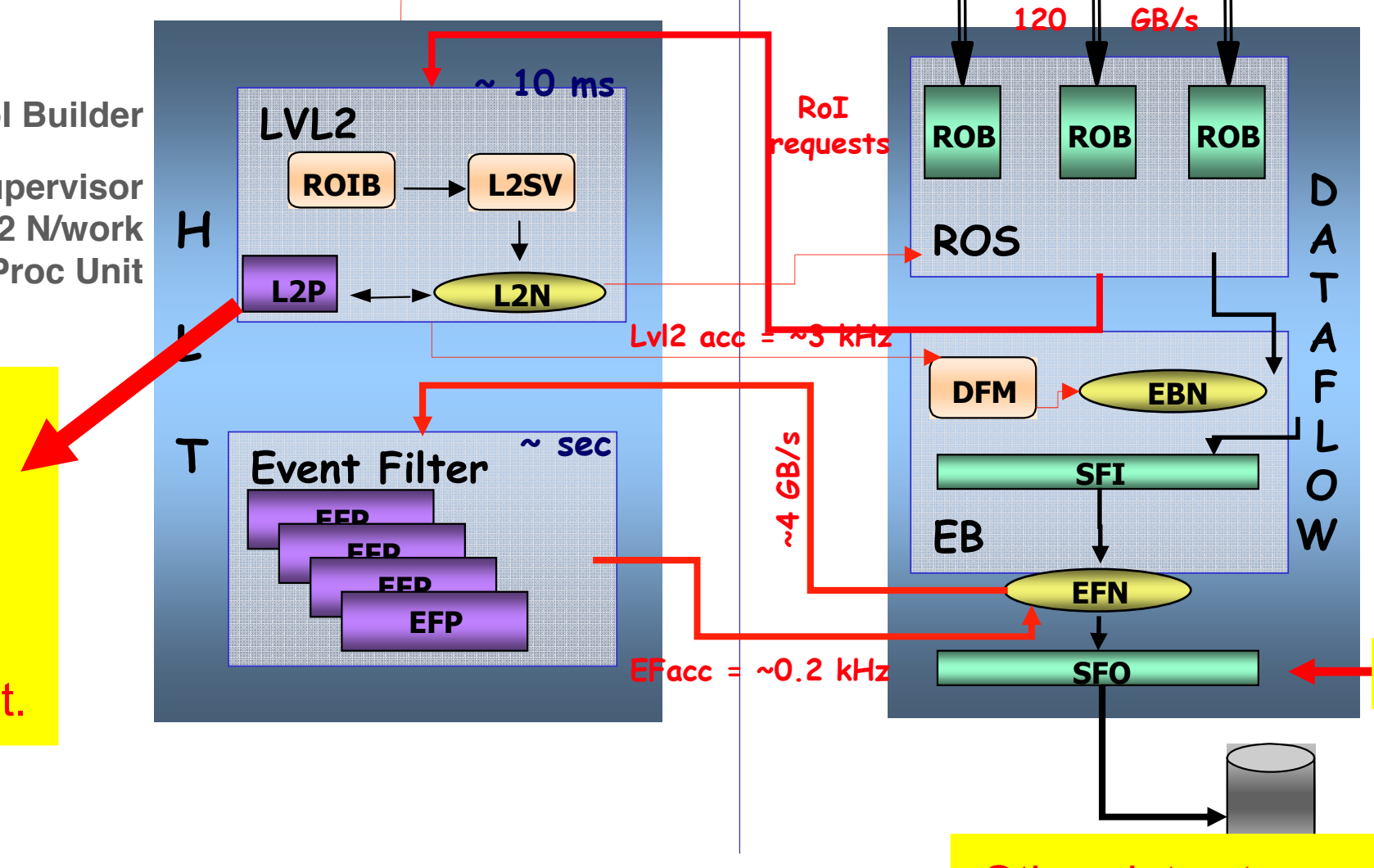

Read-Out Drivers

Read-Out Links 120 GB/s

Read-Out Buffers

Rol Builder

L2 Supervisor

L2 N/work

L2 Proc Unit

$\sim 3 \mathrm{kHz}$

New data stream for MDT

calibration and similar requirement.

$\sim 200 \mathrm{~Hz}$

Dataflow Manager $6 B / s$

Event Building N/work

Sub-Farm Input

Event Builder

Event Filter N/work

Sub-Farm Output

Other data streams. 


\section{Tier Structure of ATLAS Computing Model}

Event Filter Farm at CERN

- Located near the Experiment, assembles data into a stream to the Tier 0 Center Tier 0 Center at CERN

- Raw data $\rightarrow$ Mass storage at CERN and to Tier 1 centers

- Swift production of Event Summary Data (ESD) and Analysis Object Data (AOD)

- Ship ESD, AOD to Tier 1 centers $\rightarrow$ Mass storage at CERN

Tier 1 Centers distributed worldwide (10 centers)

- Re-reconstruction of raw data, producing new ESD, AOD

- Scheduled, group access to full ESD and AOD

Tier 2 Centers distributed worldwide (approximately 30 centers)

- Monte Carlo Simulation, producing ESD, AOD. Move ESD, AOD $\rightarrow$ Tier 1 centers

- On demand user physics analysis

CERN Analysis Facility

- Analysis with heightened access to ESD and RAW/calibration data on demand Tier 3 Centers distributed worldwide

- Physics analysis 


\section{Data Processing Stages}

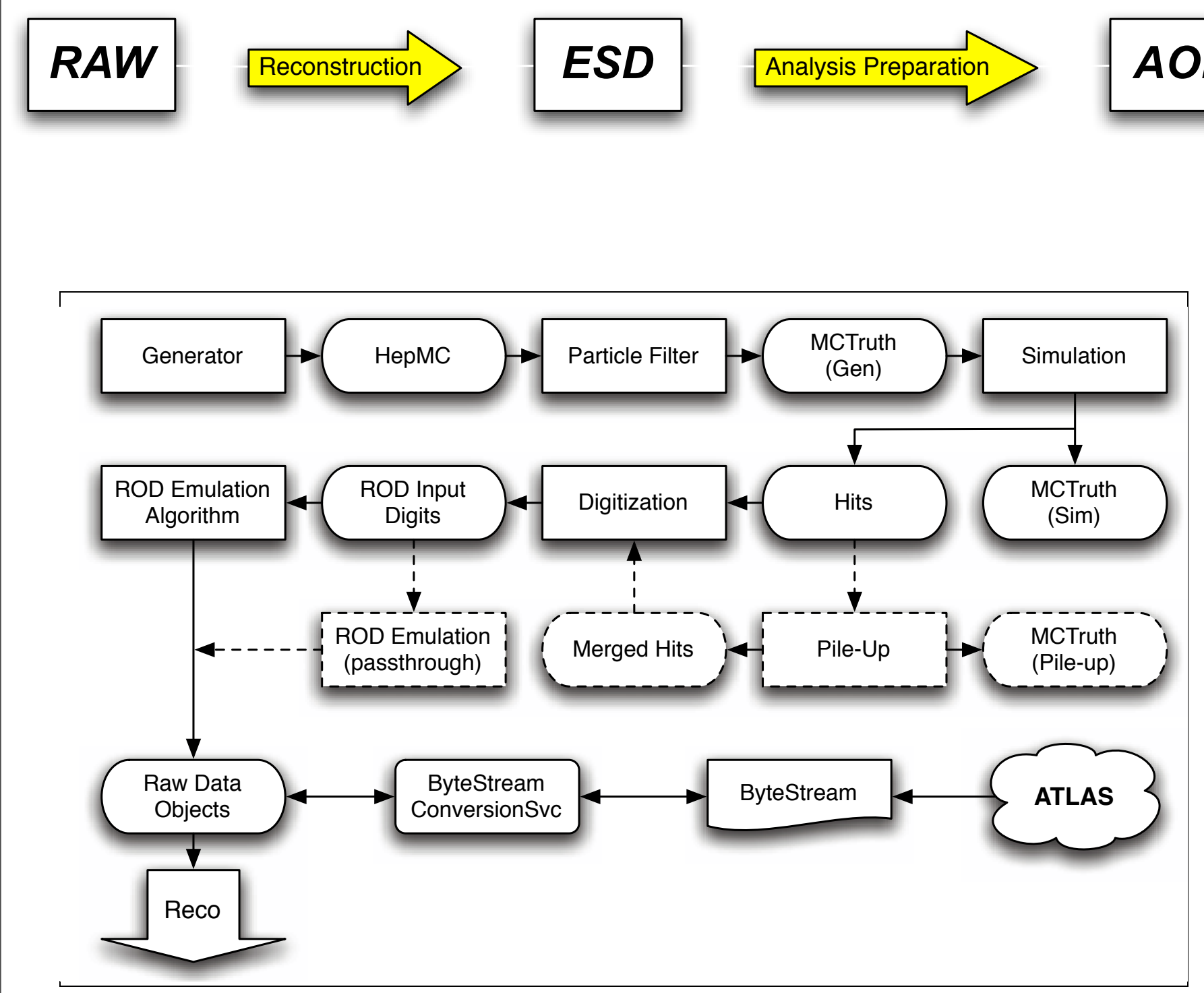

Figure 3-5 The simulation data flow. Rectangles represent processing stages and rounded rectangles represent objects within the event data model. Pile-up and ROD emulation are optional processing stages. 


\section{Distributed Data Management}

In 2004, ATLAS held a Data Challenge associated with a physics workshop

- It was the first time ESD \& AOD were used for analysis

- access to AOD was one of the main challenges

Based on this experience, ATLAS created a Distributed Data management (DDM) system: DQ2

- The scope includes the management of file-based data of all types

A major component to the design is a dataset

- composed of several files each with logical name, unique ID

- supports hierarchical datasets: datasets made from other datasets

- supports versioning

Throughput (MB/s)

Emphasis on monitoring

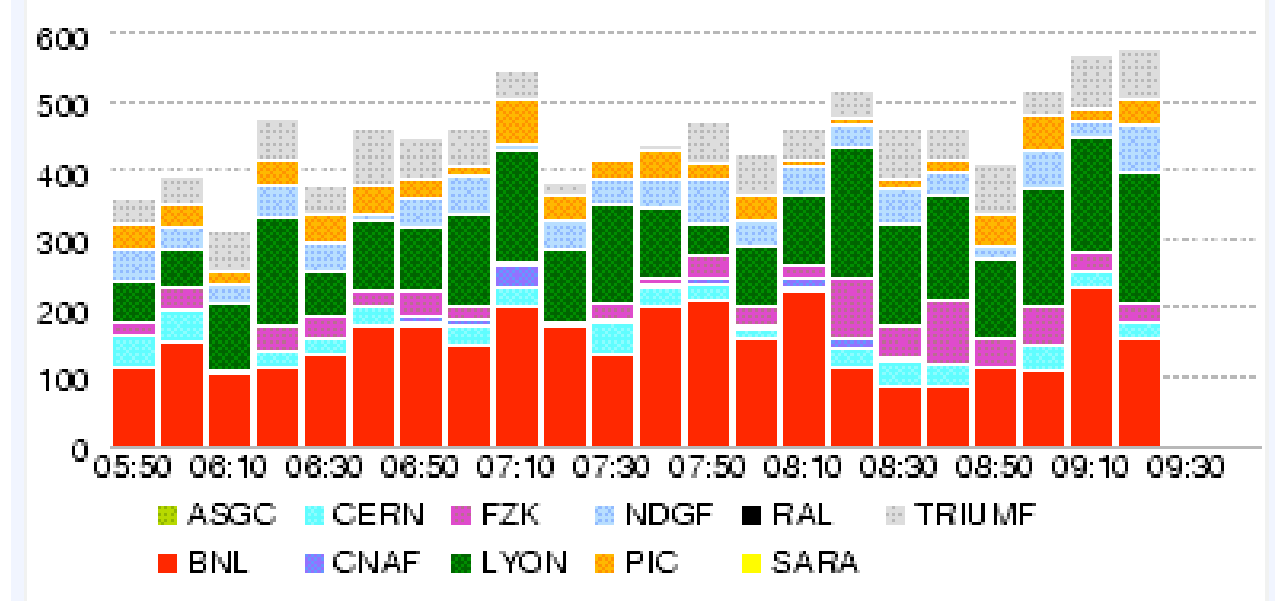




\section{Major Architectural Elements}

The Athena framework is an enhanced version of the Gaudi framework that was originally developed by the $\mathrm{LHCb}$ experiment, but is now a common ATLAS-LHCb project.

- Major design principles are:

- the clear separation of data and algorithms,

- and between transient (in memory) and persistent (on disk) data.

All levels of processing of ATLAS data, from high-level trigger to event simulation, reconstruction and analysis, take place within the Athena framework.

Packages dependencies and building is managed by CMT

POOL is used to write output of reconstruction 


\section{Gaudi: Algorithms, Tools, \& Services}

Algorithms:

- initialize(), execute(), finalize()

- retrieve or record data to the StoreGate service

- use AlgTools

AlgTools:

- interface customized for a particular action

- eg. execute(Electron)

- Produced on demand from ToolService

- Can be public or private for a particular algorithm

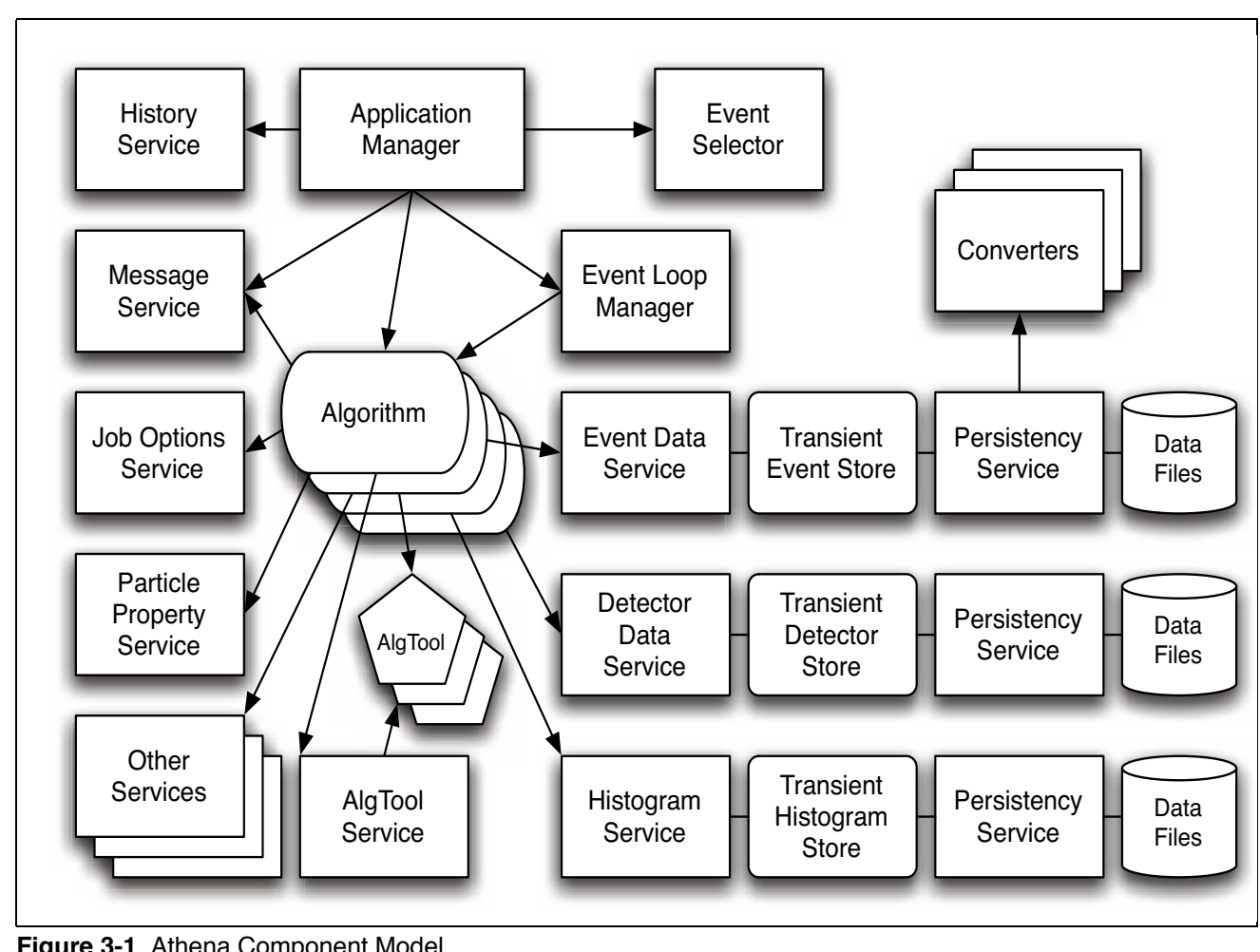

Services:

- StoreGateSvc is our blackboard for data, manages memory

- ToolSvc is a factory for AlgTools

- other services for histograming, data conversion, etc... 


\section{Python Based Configuration}

Algorithms and AlgTools can declare private data members as properties which can be modified externally from Python

- Currently undergoing a migration to Configurables

- Auto-generated Python classes encapsulate properties of $\mathrm{C}++$ classes

\section{Python}

C++

\begin{tabular}{|c|c|c|}
\hline $\begin{array}{c}\text { Configurable: } \\
\text { ElectronBuilder }\end{array}$ & automatically & $\begin{array}{c}\text { Algorithm: } \\
\text { generated }\end{array}$ \\
ElectronBuilder
\end{tabular}

\begin{tabular}{|c|c|c|}
\hline $\begin{array}{c}\text { Configurable: } \\
\text { EMShower }\end{array}$ & $\begin{array}{c}\text { automatically } \\
\text { generated }\end{array}$ & $\begin{array}{c}\text { AlgTool: } \\
\text { EMShower }\end{array}$ \\
\hline $\begin{array}{c}\text { Configurable: } \\
\text { ElectronID }\end{array}$ & $\begin{array}{c}\text { automatically } \\
\text { generated }\end{array}$ & $\begin{array}{c}\text { AlgTool: } \\
\text { ElectronID }\end{array}$ \\
\hline
\end{tabular}




\section{Python Based Configuration}

Algorithms and AlgTools can declare private data members as properties which can be modified externally from Python

- Currently undergoing a migration to Configurables

- Auto-generated Python classes encapsulate properties of $\mathrm{C}++$ classes

- Also forming higher-level abstractions in Python

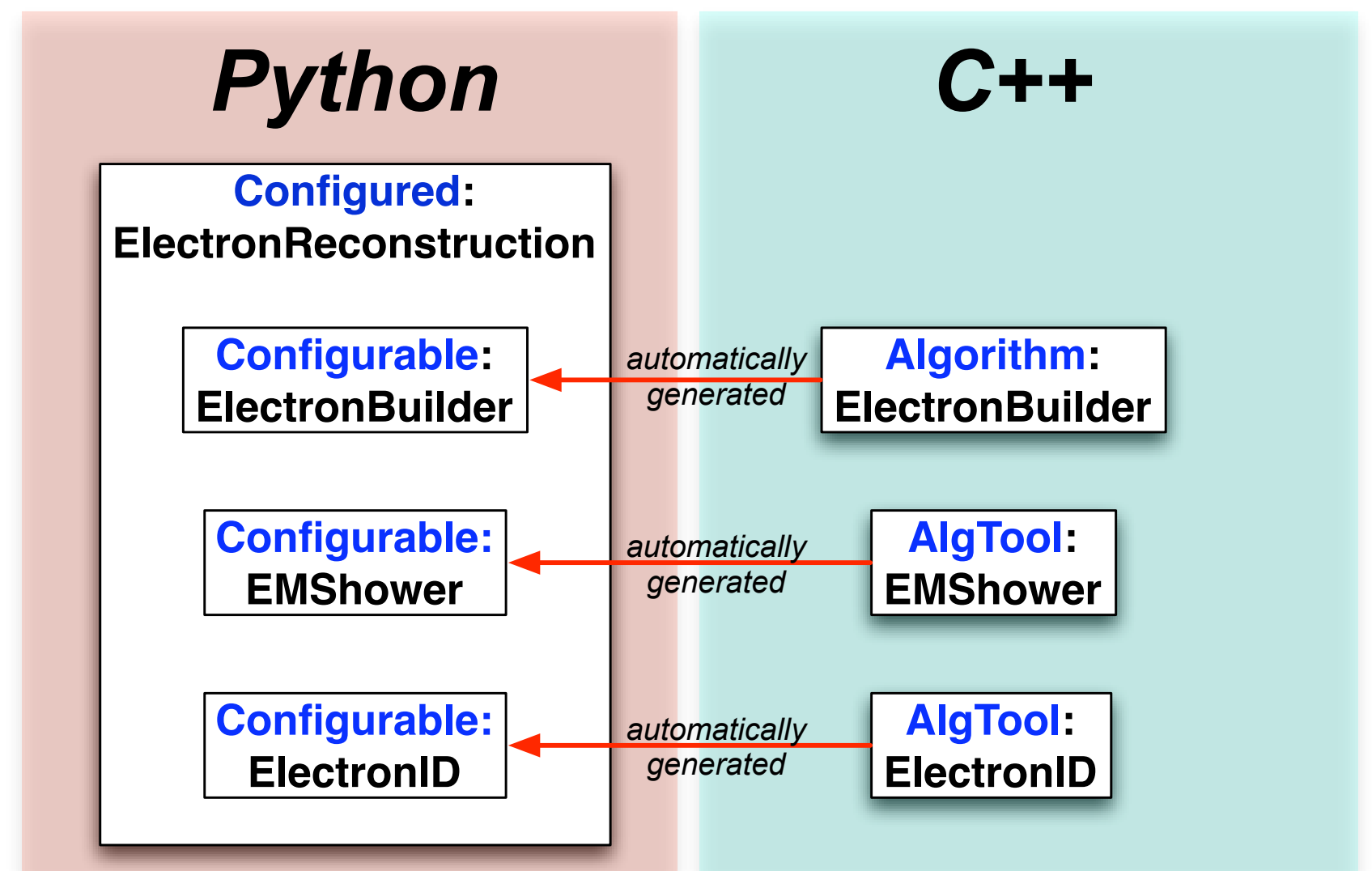




\section{Event Data Model}

One of the major design principles of Athena is a clear separation of data and algorithms.

- A flexible design of the Event Data Model (EDM) is vital!

- same EDM class often used by multiple reconstruction algorithms

- emphasis on common interfaces

Links between objects implemented with ElementLink and ElementLinkVector

- Acts as a pointer, easy to read/write

- Uses StoreGate to retrieve data

- Supports "read-on-demand"

- target data can be in another file!
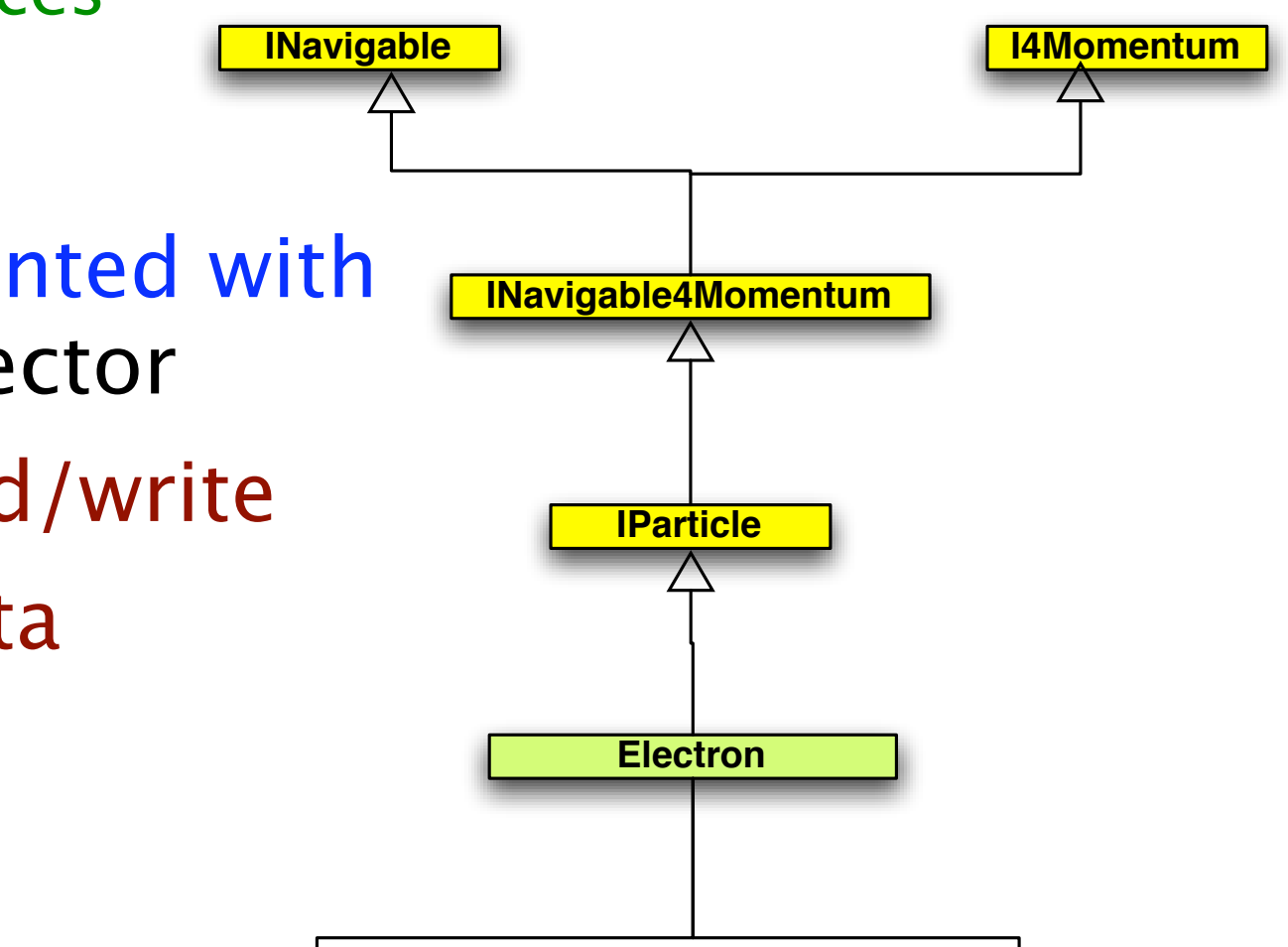


\section{Constituent Navigation}

A common feature of our EDM classes is the ability to Navigate from an object to its constituents

- Relational structure is decoupled from physical location in the files!

\section{CompositeParticle}

Clusters

Track 


\section{Constituent Navigation}

A common feature of our EDM classes is the ability to Navigate from an object to its constituents

- Relational structure is decoupled from physical location in the files!

The ESD will have cells, clusters, \& tracks

\section{CompositeParticle}

\section{Clusters}

Track

\section{Electron}

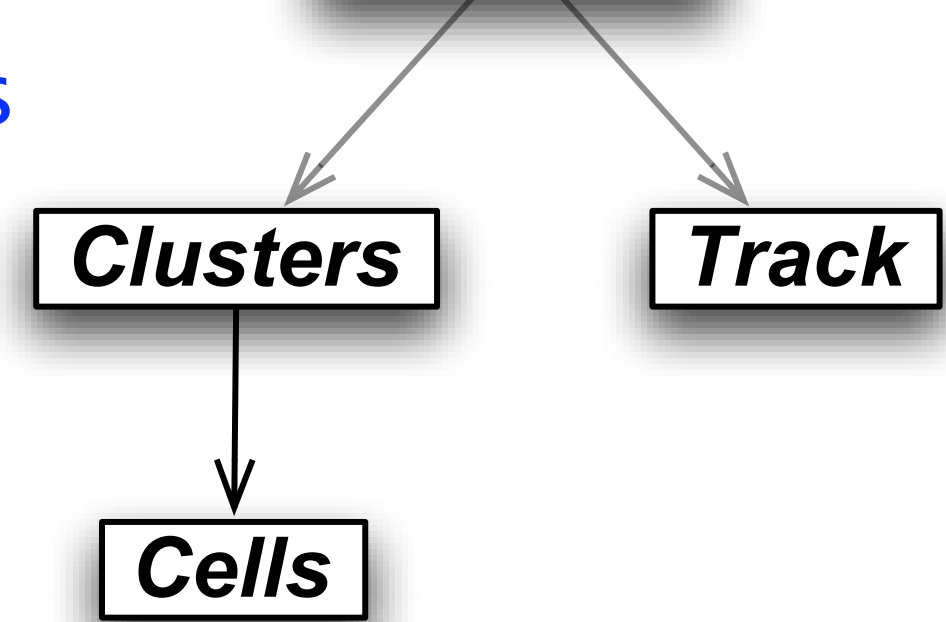




\section{Constituent Navigation}

A common feature of our EDM classes is the ability to Navigate from an object to its constituents

- Relational structure is decoupled from physical location in the files!

The ESD will have cells, clusters, \& tracks

The AOD will have electrons, clusters \&

Clusters

Electron tracks, but will not have the cells

\section{CompositeParticle}

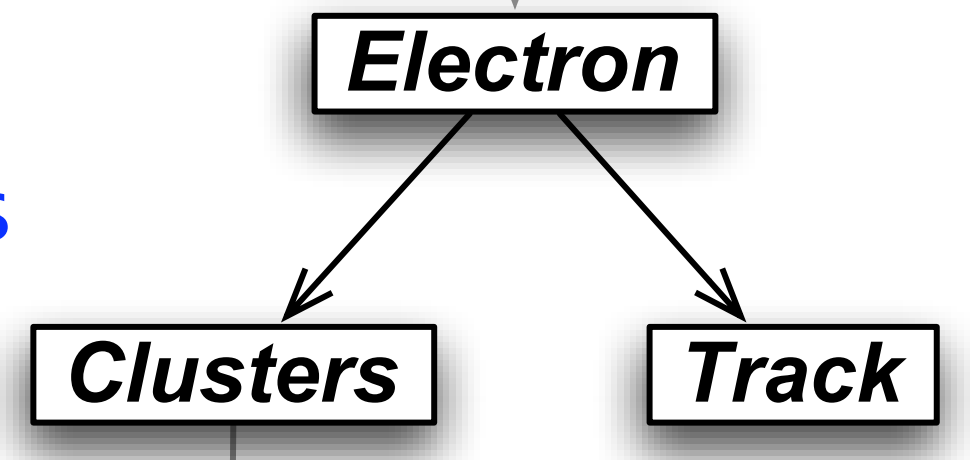

Cells 


\section{Constituent Navigation}

A common feature of our EDM classes is the ability to Navigate from an object to its constituents

- Relational structure is decoupled from physical location in the files!

The ESD will have cells, clusters, \& tracks

The AOD will have electrons, clusters \&

\section{Clusters} tracks, but will not have the cells

The DPD may have a composite particle and the electrons it was made from, but

\section{CompositeParticle} not store the clusters or track

It is possible to navigate from Composite Particle to cells if one has access to ESD \& AOD

- navigating between files called "Back Navigation" 


\section{Skimming, Thinning, and Slimming}

We have identified several complementary strategies for reducing and refining information from RAW to four-vectors

Skimming: selecting only interesting events based on TAG

Thinning: selecting only interesting objects from a container

Slimming: selecting only interesting properties of an object

Each of these data reduction strategies is highly configurable - allows the user to customize content for their purpose - does not change underlying event data model 


\section{AOD/ESD Merger and Slimming}

Initially, AOD \& ESD had different representations of particles

- needed to reduce information from ESD to AOD

- we were unable to run AOD-based algorithm on ESD

Recently, we merged AOD \& ESD representations

- outsource most of information to external objects

User can choose what information is needed for their analysis

- an example of "slimming"

\section{$E S D$}

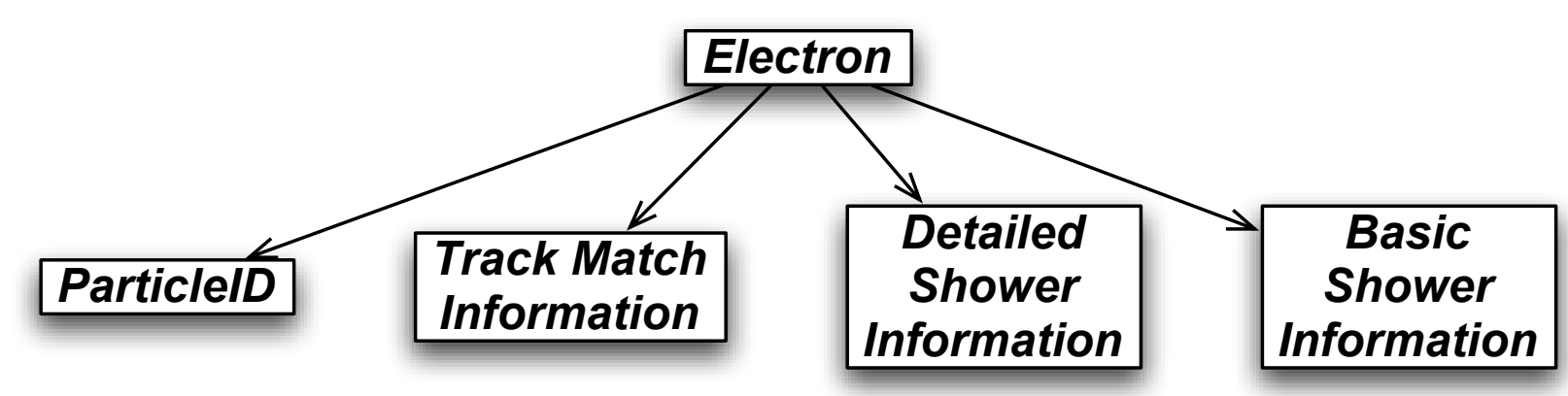




\section{AOD/ESD Merger and Slimming}

Initially, AOD \& ESD had different representations of particles

- needed to reduce information from ESD to AOD

- we were unable to run AOD-based algorithm on ESD

Recently, we merged AOD \& ESD representations

- outsource most of information to external objects

User can choose what information is needed for their analysis

- an example of "slimming"

\section{$A O D$}

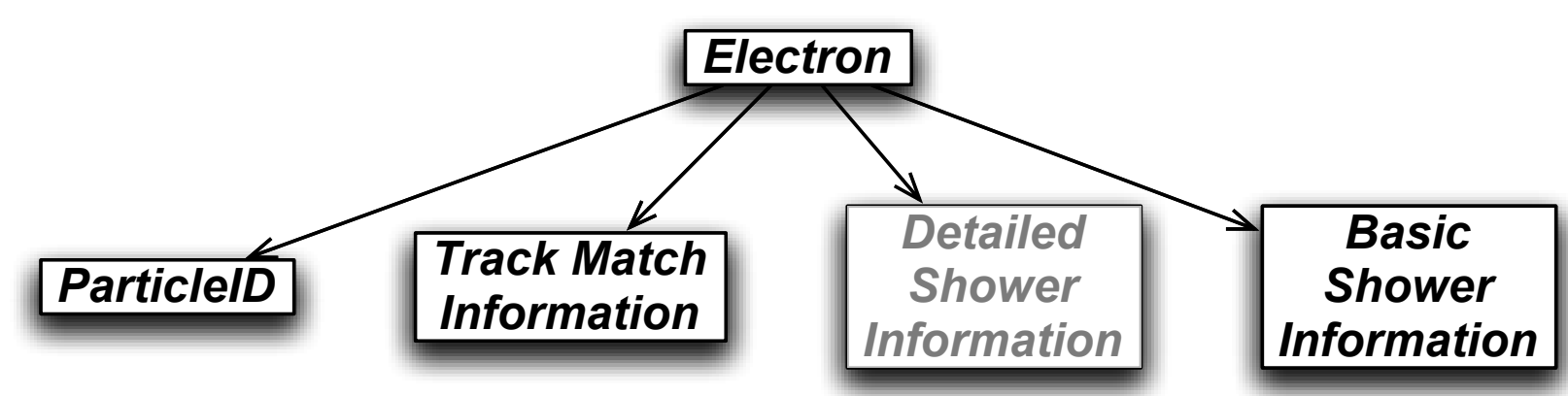




\section{AOD/ESD Merger and Slimming}

Initially, AOD \& ESD had different representations of particles

- needed to reduce information from ESD to AOD

- we were unable to run AOD-based algorithm on ESD

Recently, we merged AOD \& ESD representations

- outsource most of information to external objects

User can choose what information is needed for their analysis

- an example of "slimming"

\section{DPD}

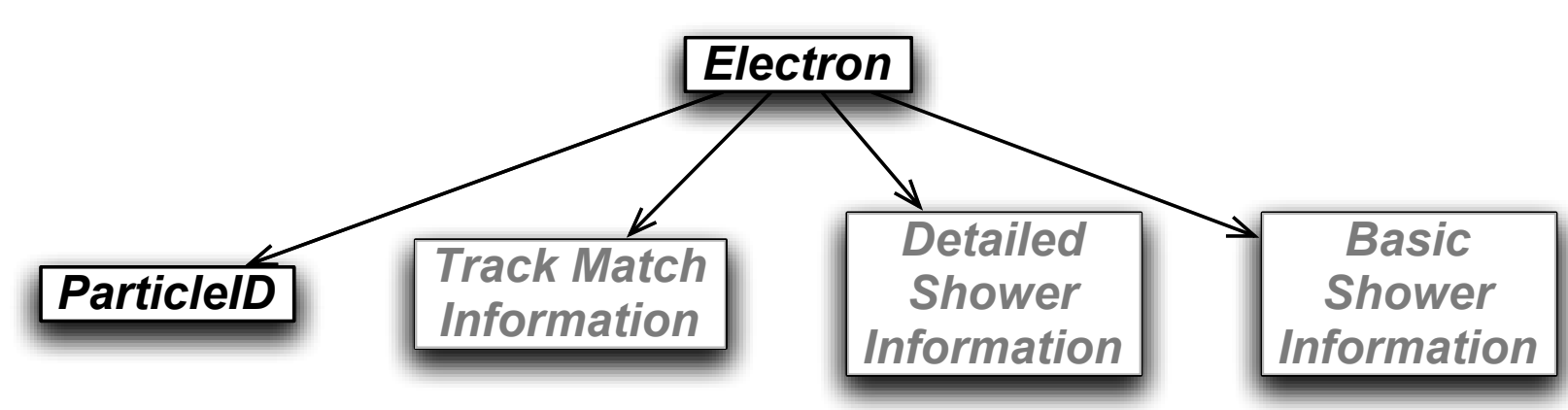




\section{TAGs \& Athena-Aware Ntuples}

ATLAS will produce event-level metadata called TAGs

- simple quantities for selecting interesting events

- can be stored in database or as a ROOT TTree.

- TAGs will span across AOD streams

- target size is $1 \mathrm{kB} /$ event

The output of Athena analysis is often an ntuple

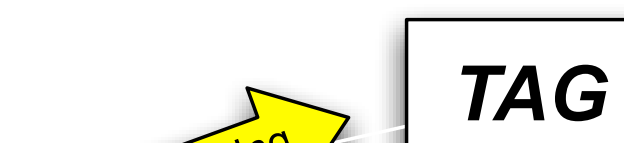

$A O D$

DPD

- our most common format for Derived Physics Data (DPD)

- recently we added hooks to make them "Athena-Aware"

- allows us to use these ntuples like they are TAGs 


\section{Derived Physics Data (DPD)}

\section{RAW Reconstruction $E S D$ Analysis Preparation $A O D$}

The AOD is the last common, centrally produced format

Analysis on AOD produces Derived Physics Data (DPD)

- contents will be analysis specific

The most common DPD format now is an Athena-Aware ntuple

- simple "flat" structure easily accessible in ROOT

- can be used for skimming, but can't process in Athena Recent attention on improving the DPD format

- add structure to DPD (eg. add Electron object)

- write DPD with POOL so that we can process it with Athena 


\section{Transient-Persistent Separation}

ATLAS has recently made a separation between the Transient (in memory) and Persistent (on disk) representation of the data

- Allows us to drastically change transient model and continue to read old data (ie. schema evolution)

Previously, we used POOL to write the transient class directly

Transient (in memory)

- complex data structure was quite slow to read $\&$ write

\section{Electron}

- typically I/O: 2MB/s

Now the persistent model can be highly optimized to reduce space and improve $\mathrm{I} / \mathrm{O}$ performance

- now read $\sim 10 \mathrm{MB} / \mathrm{s}$

\section{Electron_p1}

- size on disk reduced $\times 1-10$

Persistent (on disk) 


\section{ROOT Interoperability}

ATLAS currently has a multi-stage analysis model

- Jet finding, B-tagging, etc. happen in Athena

- Final plots, cut optimization, etc. happen in ROOT

The transition between Athena and ROOT depends on

- requirements of the analysis

- personal preferences

- maturity of the analysis

Recently we have developed technology to use ESD/AOD/ DPD written with POOL directly in ROOT

- allows us to unify file formats and save disk space

- port code between ROOT and Athena

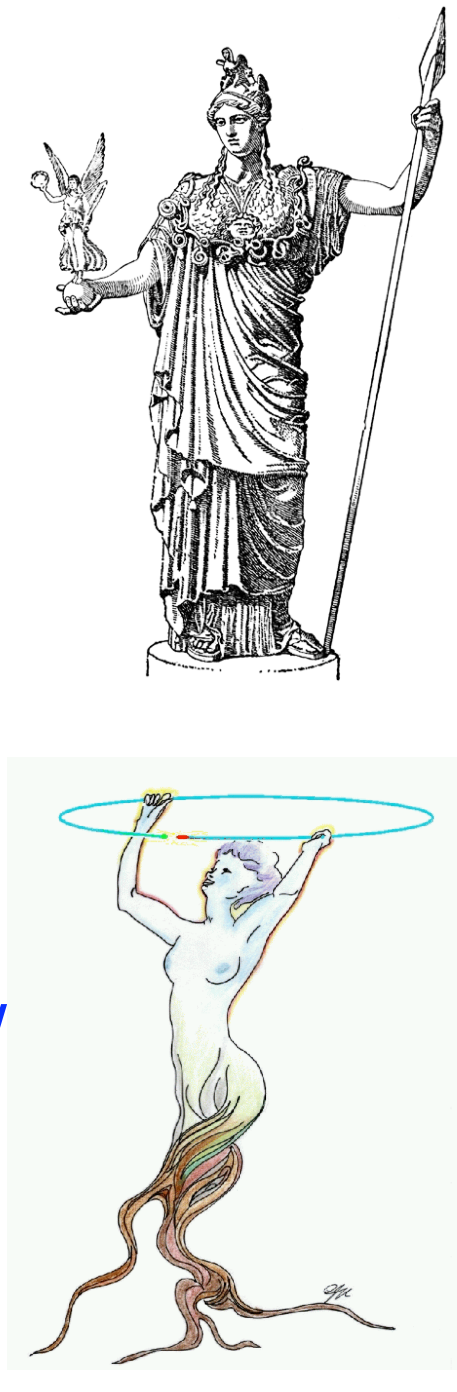




\section{Interactive Athena}

In addition to improving ROOT interoperability, we can use Athena for interactive studies

- Dictionaries and Python bindings are generated for all EDM classes

From the Athena (python) command prompt one can:

- access any object in StoreGate (eg. Electrons) for an event

- make plots looping over several events

One can even write an analysis algorithm in Python

- acts as a normal algorithm in the Gaudi/Athena framework 


\section{Distributed Analysis Tools}

Distributed analysis tools take advantage of Grid resources for processing.

- Unlike Monte Carlo production and reconstruction processing tasks, analysis is very "chaotic"

Two main tools for distributed analysis GANGA and pAthena

- Both tools provide a user interface similar to local running

- local: athena myjobOptions.py mylnput.py

- grid: pathena mylobOptions.py -inDS ttbar.AOD --outDS user.KyleCranmer.myAnalysis.ttbar

- Both tools provide bookkeeping and are interfaced with Distributed Data Management tools (eg. DQ2)

- output is registered as a DQ2 dataset

- can be used as an input to subsequent jobs

Already several hundred users!

- both tools developing quickly with significant user feedback 


\section{EventView}

We have developed an analysis framework in Athena based on EventView

The EventView is an EDM class that:

- represents a consistent interpretation of an event (particle ID, combinatorics, etc.)

- points to particles in AOD/ESD with labels

- holds UserData for a given "View"

Very modular set of analysis tools

- easy to extend

- promotes sharing of code

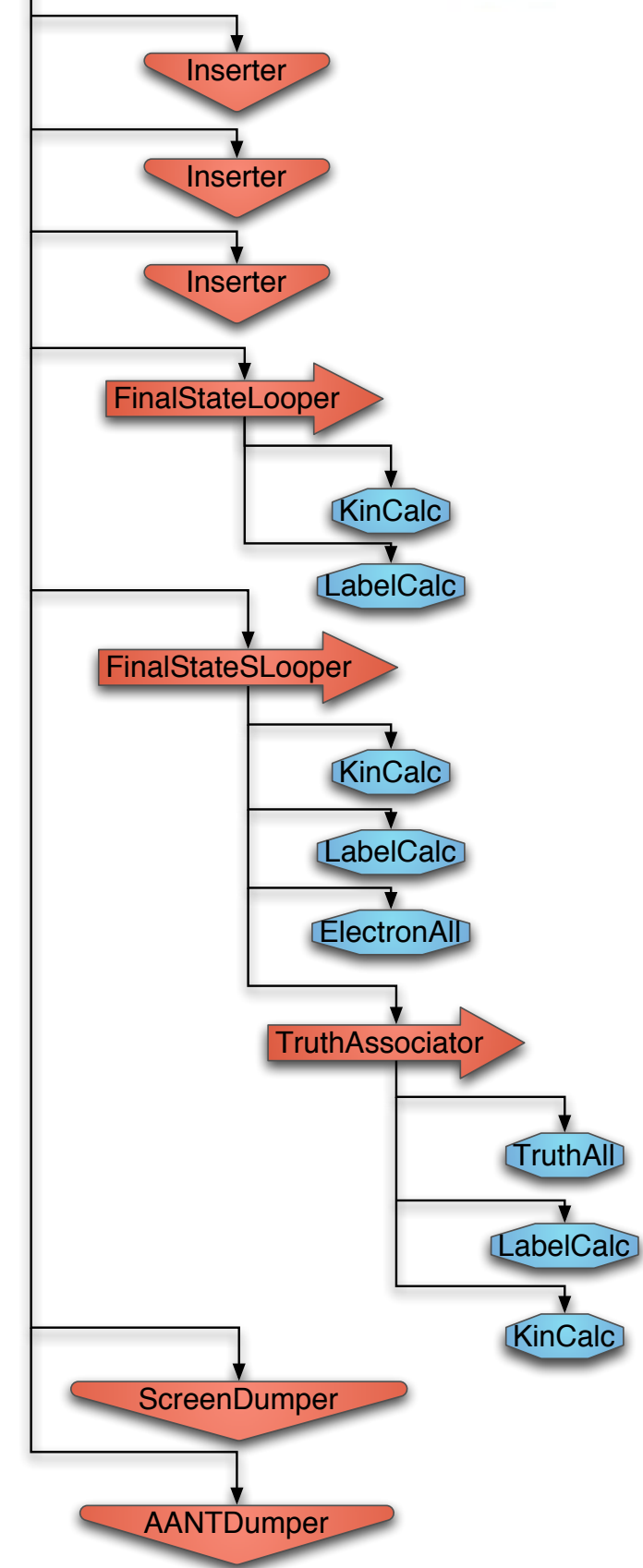




\section{Trigger-Aware Analysis}

The Atlas High-Level Trigger also runs Athena

- allows for some offline code to be re-used

As a result, our trigger simulation can use exactly the same algorithms as online

Trigger objects are stored in ESD \& AOD

- we can study trigger menus by reruning trigger with different thresholds

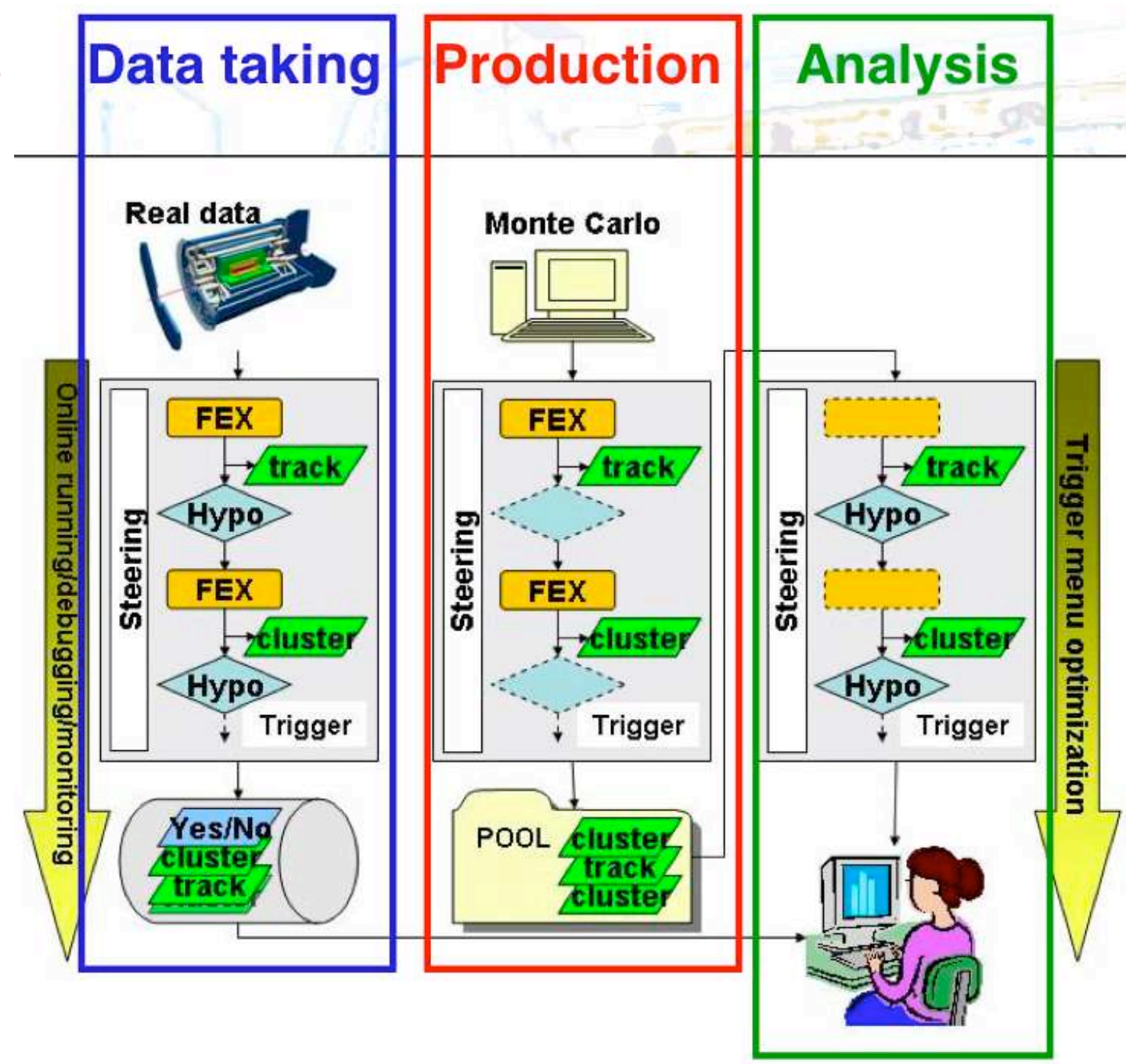

- both real \& simulated data 


\section{Event Display}

ATLAS has 3 major displays

- Atlantis: 2D views based on ALEPH's DALI display, external Java application

- Persint: specific to muons

- v-Atlas: runs within Athena, supports extensions by user

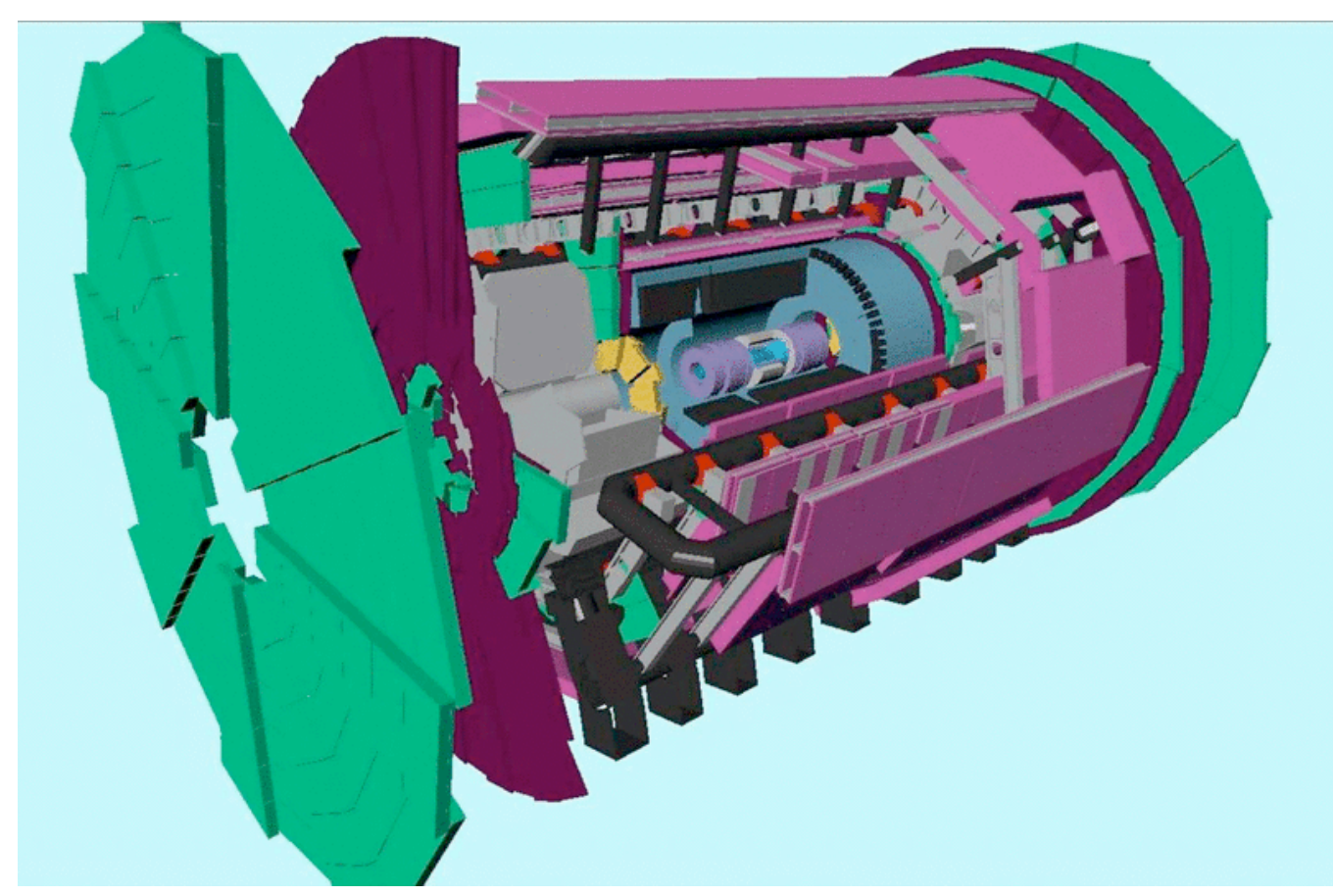

\section{ATLAS detector

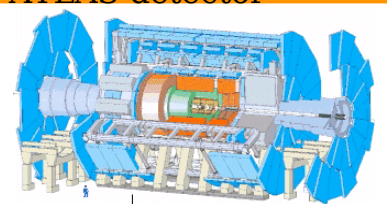

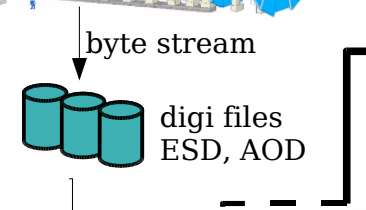
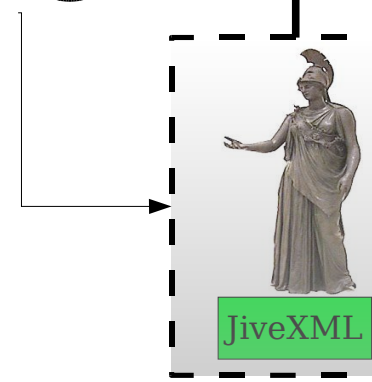

offline framework Athena runs recostruction chain and JiveXML converter
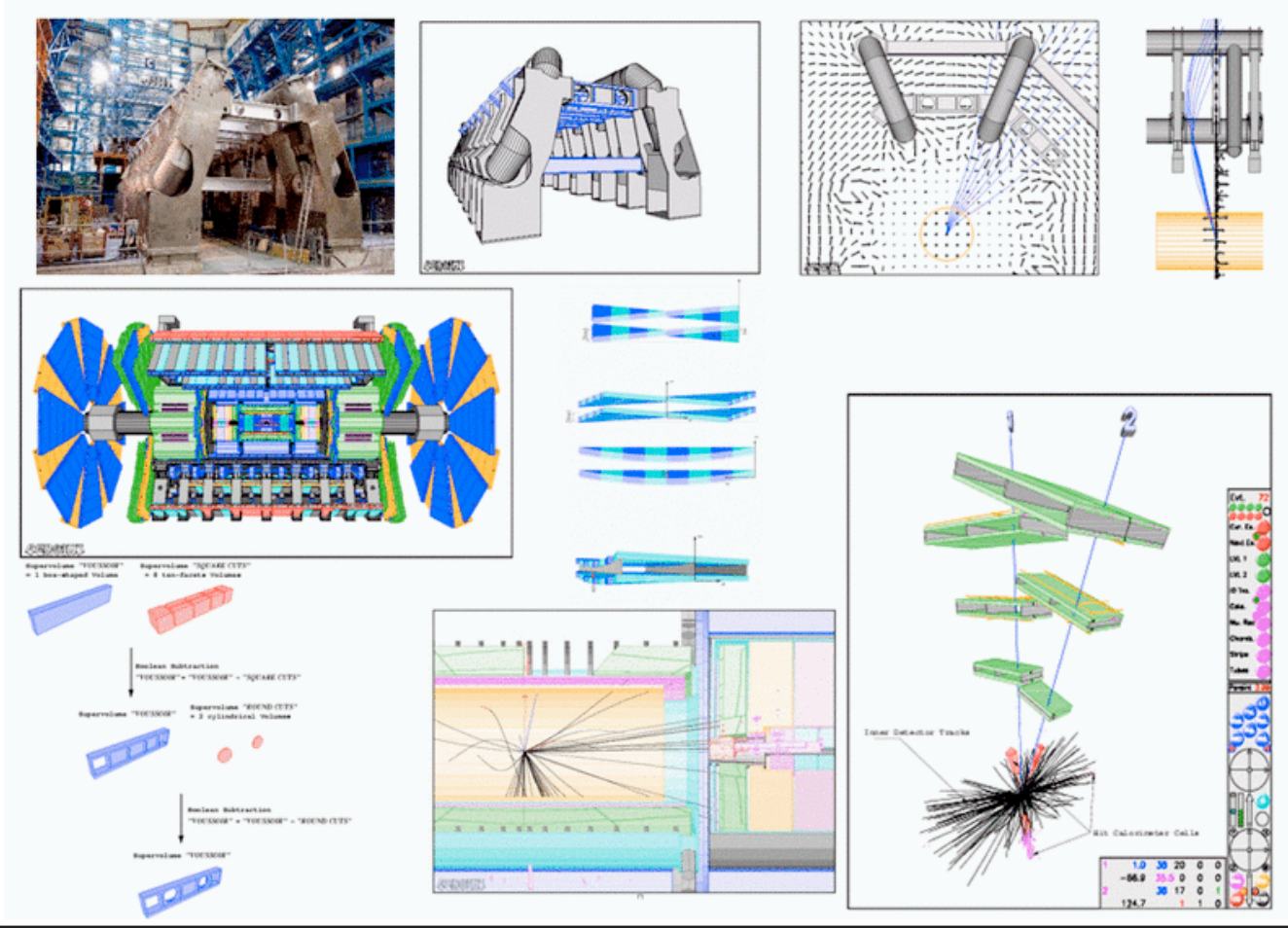


\section{Conclusions}

The ATLAS Analysis Architecture has matured significantly in the last few years.

- Most analyses based on AOD \& ESD written with POOL

- significant feedback in the last few years has led to an evolution of the analysis model and feed back to the computing model

- We have a strong framework with clear design principles

- including several strategies for data reduction

- common analysis tools with a large user-community

- Recent developments in the format of Derived Physics Data

- DPD that can be used in both Athena and ROOT

- Distributed data management and distributed analysis are also maturing based on feedback from real users

ATLAS is looking forward to the LHC startup! 


\section{Backup}

Kyle Cranmer (BNL)

HCP, Isola d'Elba, March 23, 2007 
Table 2-2 The assumed event data sizes for various formats, the corresponding processing times and related operational parameters.

\begin{tabular}{llr}
\hline Item & Unit & Value \\
\hline Raw Data Size & MB & 1.6 \\
ESD Size & MB & 0.5 \\
AOD Size & kB & 100 \\
TAG Size & kB & 1 \\
Simulated Data Size & MB & 2.0 \\
Simulated ESD Size & MB & 0.5 \\
Time for Reconstruction (1 ev) & kSI2k-sec & 15 \\
Time for Simulation (1 ev) & kSI2k-sec & 100 \\
Time for Analysis (1 ev) & kSI2k-sec & 0.5 \\
Event rate after EF & Hz & 200 \\
Operation time & seconds/day & 50000 \\
Operation time & days/year & 200 \\
Operation time (2007) & days/year & 50 \\
Event statistics & events/day & $10^{7}$ \\
Event statistics (from 2008 onwards) & events/year & $2 \cdot 10^{9}$
\end{tabular}

